\title{
A GRAVITATIONAL LENS CANDIDATE BEHIND THE FORNAX DWARF SPHEROIDAL GALAXY
}

\author{
C.G. TINNEY \\ Anglo-Australian Observatory \\ PO Box 296, Epping. NSW. 2121 Australia.
}

CCD astrometry makes possible milli-arcsecond precision relative astrometry at faint magnitudes (Tinney 1994a). The measurement of proper motions for the nearest galaxies is therefore a project requiring only years - not decades. A prerequisite for such measurements, however, is a set of unresolved, extragalactic reference objects - ie. QSOs. While searching for such objects behind the Fornax dSph galaxy, Tinney (1995b) discovered the QJ0240-343AB system as a pair of bright, UV-excess objects (U-B $=-1.0, \mathrm{~B}=19.0 \& 19.8$ ) with a separation of $6.1^{\prime \prime}$. Spectra obtained with the $3.9 \mathrm{~m}$ Anglo-Australian telescope and the RGO+FORS spectrographs (Fig. 1) have shown both components of the pair to be at a redshift of $z=1.4$, with a rest velocity difference consistent with zero at a 1- $\sigma$ limit of $180 \mathrm{~km} / \mathrm{s}$. The spectra also show a definite metal-line absorption system at $z=0.543$ and a possible system at $z=0.337$. No bright lens is seen, but the strong similarities in the spectra suggest a gravitational lens nature.

If the system is a lens, then the large difference in the line-to-continuumratio of the components suggests either strong variability, or microlensing of the continuum source. A search has been made of the literature and astronomical archives for images of the QJ0240-343AB pair - the data obtained to date are shown in Table 1. (The data from the NTT is in the $\mathrm{R}$ band, but non-photometric. Stars in the field have been used to create a uniform, but arbitrary, zero-point. The zero-point of the UKST data is very uncertain.) It seems clear from this data that the system is genuinely variable, on time scales of a few years (the time scale for micro-lensing should be $\sim 30$ years). The existing data does not enable statements about time delays to be made, but suggest that monitoring will straightforwardly provide much information on this system. 


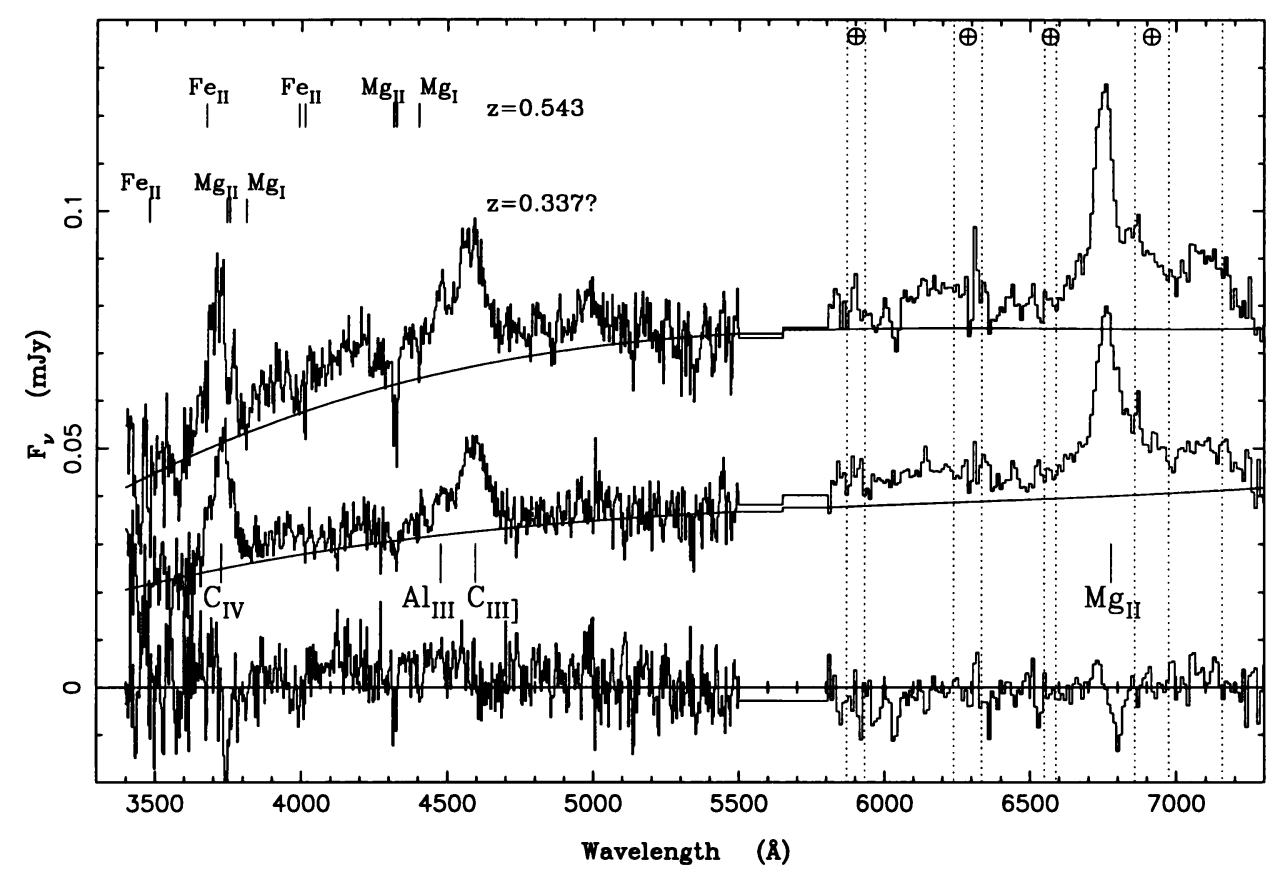

Figure 1. Spectra obtained with the 3.9m AAT and RGO+FORS of QJ0240-343A (upper trace) and QJ0240-343B (middle trace), and the difference between the line spectra (lower trace) after the subtraction of a continuum. Notice that the lines have roughly equal strength in the two components, while the continuum of $\mathrm{A}$ is twice that of $\mathrm{B}$.

TABLE 1. QJ0240-343AB Photometry

\begin{tabular}{lrcccc}
\hline Source & Epoch & $\mathrm{A}$ & $\mathrm{B}$ & Unc & $\mathrm{A}-\mathrm{B}$ \\
\hline Demers et al. 1994 & 20 Dec 1974 & $\mathrm{B}_{j}=19.72$ & $\mathrm{~B}_{j}=19.97$ & \pm 0.1 & $\Delta \mathrm{B}_{j}=-0.38$ \\
& 20 Dec 1974 & $\mathrm{V}=19.71$ & $\mathrm{~V}=20.43$ & \pm 0.1 & $\mathrm{~V}=-0.72$ \\
UKST/COSMOS & 16 Nov 1979 & $\mathrm{B}_{j}=17.5$ & $\mathrm{~B}_{j}=17.5$ & $\pm 0.2)$ & $\Delta \mathrm{B}_{j}=0.0$ \\
ESO/NTT Archive & 5 Aug 1991 & $\mathrm{R}_{91}=19.95$ & $\mathrm{R}_{91}=20.54$ & \pm 0.03 & $\Delta \mathrm{R}_{91}=-0.59$ \\
ESO/NTT Archive & 24 Oct 1992 & $\mathrm{R}_{91}=19.94$ & $\mathrm{R}_{91}=20.35$ & \pm 0.03 & $\Delta \mathrm{R}_{91}=-0.41$ \\
Tinney 1995 & 27 Oct 1994 & $\mathrm{B}=19.00$ & $\mathrm{~B}=19.77$ & \pm 0.05 & $\Delta \mathrm{B}=-0.77$ \\
\hline
\end{tabular}

\section{References}

Demers, S., Irwin, M. \& Kunkel, W. 1995, AJ, 108, 1648

Tinney, C.G. 1995a, in Science with the VLT, eds. J.R. Walsh \& I.J. Danziger, (SpringerVerlag: Heidelberg)

Tinney, C.G. 1995b, MNRAS, in press 